\title{
Time-frequency analysis of the dynamics of different vorticity structures generated from a finite-length triangular prism
}

\author{
G.V. Iungo*, E. Lombardi \\ University of Pisa, Department of Aerospace Engineering, via Caruso 8, 56122 Pisa, Italy
}

\section{A R T I C L E I N F O}

Available online 22 April 2011

Keywords:

Time-frequency analysis

Proper orthogonal decomposition

Instantaneous frequency

Singular spectrum analysis

\begin{abstract}
A B S T R A C T
Time-frequency analysis of the dynamics of different wake vorticity structures, generated from a triangular prism orientated with its apex edge against the incoming wind, is carried out. Time-frequency analysis of time-series obtained with hot-wire anemometry is performed through a procedure based on proper orthogonal decomposition and spectral components are extracted with a technique that provides an increased efficiency for fluid dynamic applications.
\end{abstract}

(c) 2011 Elsevier Ltd. All rights reserved.

\section{Introduction}

Fluid dynamic signals are generally characterized by significant fluctuations that must be characterized in order to investigate on their physical origins. In several conditions, as for instance in wakes and jets, the flow fluctuations may show dominating spectral components, which can be singled out through the conventional Fourier transform. However, this technique gives only a timeinvariant amplitude and frequency for each spectral component, thus becomes highly inappropriate for non-stationary signals.

The wavelet transform may directly be applied to multicomponent signals in order to characterize the time-variation of all the spectral components present in a certain frequency range. Component extraction may also be performed by using, for instance, the so-called wavelet ridges, as reported in Carmona et al. (1998), a technique proposed in Buresti et al. (2004) or a more sophisticated wavelet decomposition presented in Olhede and Walden (2004).

A technique widely used to detect and extract the different dynamics present in a flow is the proper orthogonal decomposition (POD in the following). POD was introduced in fluid dynamics for investigations on turbulence by Lumley (1967), who proposed a method for extraction of coherent structures from turbulent velocity fields. This method was then applied by Payne and Lumley (1967) to the large eddy simulation of the wake produced from a circular cylinder, by Bakewell and Lumley (1967) for a boundary layer survey and by Holmes and Lumley (1996) for turbulence modeling and detection of coherent structures. POD was used in Gamard et al. (2002) to analyze signals simultaneously acquired from 138

\footnotetext{
* Corresponding author. Tel.: + 39050 2217227; fax: + 390502217244 .

E-mail addresses: valerio.iungo@epfl.ch, giacomo.iungo@ing.unipi.it (G.V. Iungo).
}

hot-wires in order to investigate on an axisymmetric turbulent jet. In Perrin et al. (2008) the vortex shedding generated from a circular cylinder was surveyed with the two most energetic components detected through POD.

POD is also suitable to devise optimal lower-dimensional subspaces on which the governing equations of a dynamical system may be projected through, for instance, a Galerkin projection (see Ma and Karniadakis, 2002; Galletti et al., 2004; Buffoni et al., 2006). POD is a fundamental tool used for flow control, as in Bergmann et al. (2009) and Weller et al. (2009). A significant review on the use of POD in wind engineering, together with some applications (e.g. for atmospheric flows over different topographies, analysis of the aerodynamic forces acting on a tall building model, buffeting of a long-span bridge), is reported in Solari et al. (2007) and its companion paper (Carassale et al., 2007).

By considering time-frequency analysis of single-point measurements, i.e. time-series, a technique based on POD for component detection and extraction is the singular-spectrum analysis (SSA). The application of this technique requires the definition of the so-called window length or embedding dimension, $N_{\text {period, }}$ which allows to vary the frequency resolution of the time-frequency analysis. Since $N_{\text {period }}$ is chosen, the eigen-decomposition of the lagged covariance matrix of the signal, which has a Toeplitz structure, is performed. As typical for all the POD-based techniques, a certain spectral component is associated to a pair of POD modes with nearly equal energy, thus for its extraction both POD modes must be used; to this end a method for detection of POD mode pairs was proposed in Vautard et al. (1992). Moreover, the principal components obtained with SSA are characterized by a reduced time-length with respect to the one of the source-signal, so that a method to remedy to this drawback was proposed in Vautard et al. (1992). In Pastur et al. (2008) an application of SSA for the analysis of the intermittency of two different spectral components present in the flow over an open cavity is reported. 
In the present paper a procedure for time-frequency analysis of time-series is presented, which is based on POD. The sourcesignal is first manipulated in order to generate an ad-hoc data set consisting of time-portions of the signal, each one composed of the same number of samples; this data set represents the input for the POD procedure. The result obtained from the POD is an orthogonal basis whose elements, denoted as POD modes, are sorted by their energy. The POD modes corresponding to higher energy represent the most significant signal fluctuations and their Fourier analysis already provides spectral information related to each POD mode. However, and more importantly, the components associated with each POD mode, with their amplitude and frequency modulations, can be obtained through a procedure based on the convolution of the source-signal with the relevant POD mode, suitably manipulated.

The present paper is organized as follows: the POD procedure for time-frequency analysis is described in Section 2 together with the technique for component extraction. The technique is then applied to hot-wire anemometry signals acquired in proximity of the wake generated from a triangular prism (Section 3). Finally, some conclusions are drawn in Section 4.

\section{POD procedure for time-frequency analysis of time-series}

POD is a method providing an orthonormal basis for the modal decomposition of an ensemble of data functions and is completely a posteriori, data dependent and does not neglect the nonlinearities of the original dynamical system, even being a linear procedure; furthermore, the POD basis is orthogonal. The most peculiar feature of POD is optimality: among all linear decompositions it provides the most efficient detection, in a certain least squares optimal sense, of the dominant components and trends of an infinite-dimensional process.

Let us consider a generic zero-mean time-series $u(t)$, which can represent a measurement performed in a fixed point of the flow field with a sampling frequency $F_{\text {samp }}$ and a total number of samples

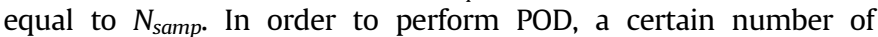
observations of the analyzed process are required, the so-called snapshots; to this end, time-portions of the source-signal $u(t)$ are generated, all with the same number of samples, $N_{\text {period, }}$ partially overlapped and uniformly distributed along its time-length. Generally, the number of snapshots, $N_{\text {snap }}$, is gradually increased through a sensitivity analysis, so that the convergence of the POD eigenvalues and of the most energetic POD modes is obtained.

A crucial task is represented by the choice of the time-length of each snapshot, i.e. the number of samples, $N_{\text {period, }}$, composing each snapshot, which is strictly related to the frequency resolution of the analysis; in other words, if $\Delta f$ is the required frequency interval between two consecutive elements of the signal power spectrum, then $N_{\text {period }}$ will be equal to the ratio between the sampling frequency and the frequency resolution, $F_{\text {samp }} / \Delta f$.

Once the required number of samples for each snapshot, $N_{\text {period, }}$, is chosen and the total number of snapshots, $N_{\text {snap }}$, is fixed, a matrix $M$ is generated, whose rows are the time-series of the snapshots. Subsequently, the covariance matrix of $M$ is evaluated, which is Hermitian symmetric and non-negative definite, thus its eigenvalues are real and non-negative. The eigenvectors are orthogonal and are normalized through the $L_{2}$ norm $\left(=\sqrt{\sum_{i} x_{i}^{2}}\right)$ so that the eigenvalues represent the energy associated with the respective eigenvectors.

Any snapshot, i.e. each row of the matrix $M$, is represented through a linear combination of the POD modes, $\phi(j)$ :

$M_{i}=\sum_{j=1}^{N_{\text {period }}} \phi(j) a_{i}(j)$ where $a_{i}(j)$ are denoted as principal components, which are evaluated through the following projection:

$a_{i}(j)=\left\langle M_{i}, \phi(j)\right\rangle$

where $\langle\bullet, \bullet\rangle$ is the scalar product.

The computer-generated signal used to assess the POD procedure for time-frequency analysis is a stationary time-series composed of three different spectral components $\left(f_{1}=40 \mathrm{~Hz}\right.$, $f_{2}=60 \mathrm{~Hz}$ and $f_{3}=70 \mathrm{~Hz}$ ) and white noise with an energy equal to $23 \%$ of the total energy of the signal:

$y_{1}=\sin \left(2 \pi f_{1} t\right)+2 \sin \left(2 \pi f_{2} t\right)+4 \sin \left(2 \pi f_{3} t\right)+W N$

The signal is sampled with a frequency of $1 \mathrm{kHz}$. The POD procedure for time-frequency analysis is applied by using for this test-case $10^{4}$ snapshots, which represent adjacent timeportions of the signal, each comprising 501 samples, i.e. the used frequency resolution is about $2 \mathrm{~Hz}$. The eigenvalues reported in Fig. 1, which represent the fluctuating energy of the respective POD modes, are reported as percentage of the total energy of the signal, and show that just the first six POD modes are characterized by a significant fluctuating energy and, thus, they are the only POD modes to be considered for the component extraction.

Fourier power spectra of the first eight POD modes are reported in Fig. 2. The most energetic POD modes, with the same energy, are POD modes 1 and 2, which are characterized by a dominant frequency of $70 \mathrm{~Hz}$, as expected being the most energetic spectral component of $y_{1}$ characterized by this frequency. From Fig. 2 it is seen that these two POD modes are characterized by the same power spectrum, but they are in quadrature, being elements of an orthogonal basis. As already highlighted in Vautard et al. (1992) for SSA, when a periodic dynamics is present in the signal a pair of eigen-elements with nearly equal energy is detected.

The POD modes 3 and 4 are related to the component at frequency $f_{2}=60 \mathrm{~Hz}$, while 5 and 6 to the component at $f_{1}=40 \mathrm{~Hz}$; as expected they are sorted by their energy. The remaining POD modes do not show any dominant spectral component and are due to white noise, but they are not analyzed because, as shown in Fig. 1, their relative energy is negligible.

From the power spectra of the POD modes the dominant frequencies of the signal are detected and sorted by their energy; however, their contribution along the time-length of the whole source-signal is not yet determined. Then, the technique for component extraction is based on the convolution of the source signal with the considered POD mode. However, a generic POD mode is not a symmetric filter due to its initial phase and, thus,

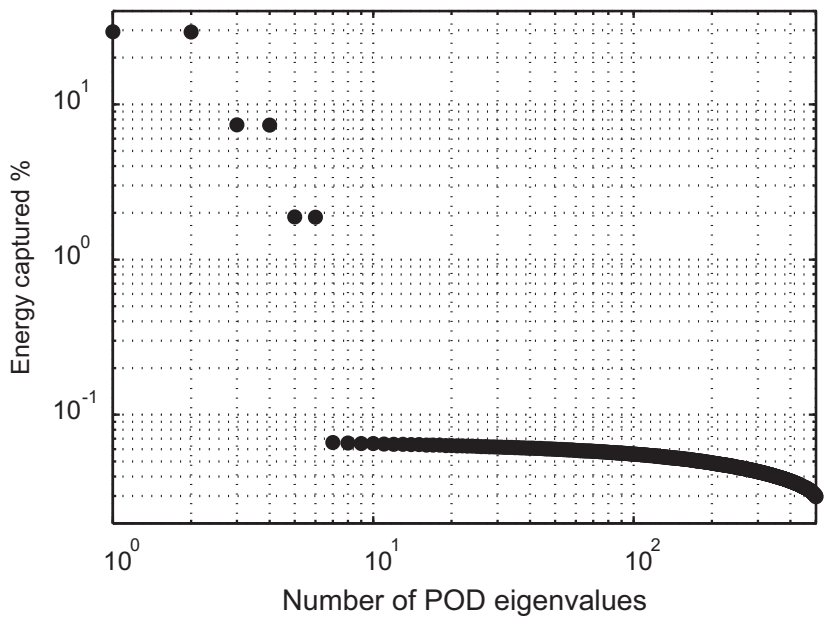

Fig. 1. POD eigenvalues evaluated for the computer-generated signal $y_{1}$. 


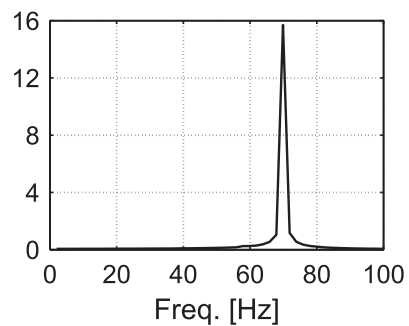

POD mode 1

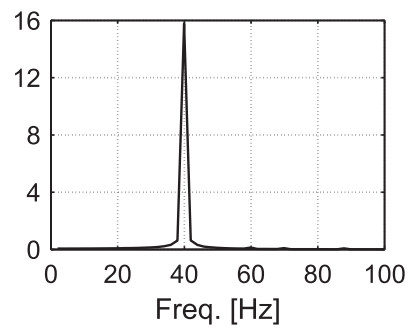

POD mode 5

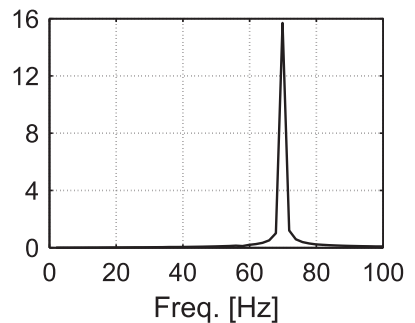

POD mode 2

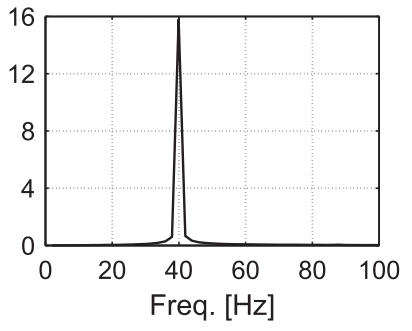

POD mode 6

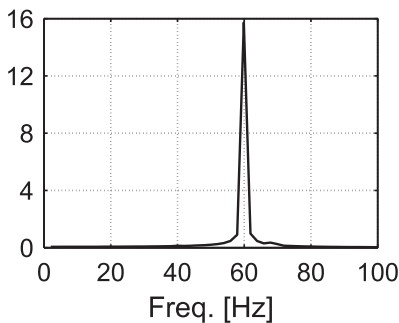

POD mode 3

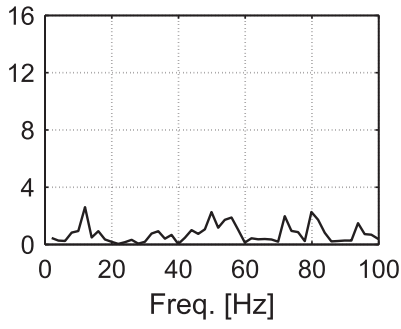

POD mode 7

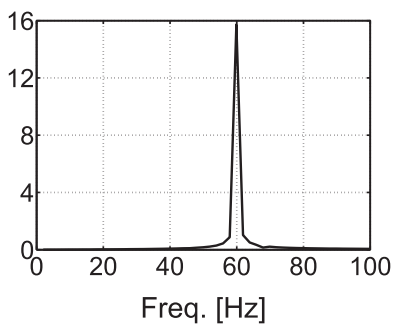

POD mode 4

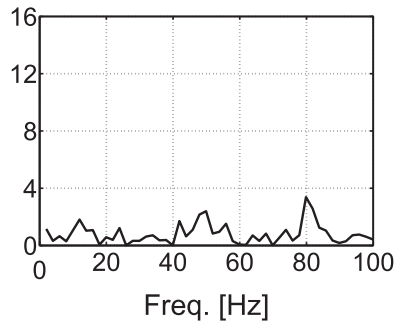

POD mode 8

Fig. 2. Fourier power spectra of the first eight normalized POD modes evaluated for the signal $y_{1}$.

the convolution produces a certain phase-shift, $\Delta \varphi$, on the extracted principal component with respect to the source-signal. In the context of signal processing, it is common to avoid this phase-shift by using as filter the reversed function, denoted as POD flip-mode. This function is composed of the elements of the considered POD mode, but from the last one backwards to the first one. Consequently, the convolution of the source-signal with the POD flip-mode produces a phase-shift equal to $-\Delta \varphi$; therefore, performing a double convolution of the source-signal, i.e. with the POD mode and with its POD flip-mode, a phasecorrection of the extracted principal component may be performed. However, a more robust procedure, and with lower computational effort, is based on performing a single convolution of the source-signal with a function obtained from the convolution of a POD mode with its respective POD flip-mode. This function, denoted as POD conv-mode, is a symmetric filter and, thus, no phase-shift is produced through the convolution of the source-signal with the POD conv-mode.

An important step regarding principal component extraction through the convolution procedure consists of avoiding any amplification or damping. The basic idea of the filtering is that the convolution of a certain POD conv-mode with itself must produce the POD conv-mode without any amplification or damping. In order to reach this goal, the result of the convolution must be normalized through the norm of the convolution of the POD conv-mode with itself, and then multiplied with the norm of the POD conv-mode in order to restore its initial energy. Therefore, the result of the convolution must be multiplied by the factor $K$ :

$K=\frac{\mid \operatorname{con} v-\text { mode } \mid}{\mid \text { convolution }(\operatorname{con} v-\text { mode,conv-mode }) \mid}$

where $|\bullet|$ represents the $L_{1}$-norm, i.e. the sum of absolute values of the elements, consistently with the precision adopted for the convolution algorithm.

As regards the computer-generated signal $y_{1}$, the three different spectral components are now extracted through the convolution of $y_{1}$ with the respective POD modes. Starting from the most energetic spectral component, i.e. the one related to $f_{3}=70 \mathrm{~Hz}$, its amplitude is equal to 4 (Eq. (3)) and through the spectral component extraction its mean value is found to be 3.996 with a standard deviation of 0.087 (about $2 \%$ of the mean value), demonstrating that the spectral contribution of interest is completely captured by this spectral component. The instantaneous frequency of the spectral component is also evaluated through the Hilbert transform and a mean value of $69.915 \mathrm{~Hz}$ and a standard deviation of 0.013 (about $0.02 \%$ of the mean value) are found.

Since the spectral component related to the POD mode 1 is extracted, a residual signal can be evaluated by subtracting the extracted spectral component from the source-signal $y_{1}$. If the spectral component related to the POD mode 2 is then extracted, it is found to correspond to a practically null signal, which can be added to the one extracted with the POD mode 1 or just neglected. This assesses that the spectral component of interest is already completely extracted by only using POD mode 1 and POD mode 2 is considered only as its coupled POD mode. This is an important feature of the present technique with respect to other possible POD-based ones; for instance, regarding SSA in Vautard et al. (1992) a method to detect pairs of eigen-elements related to a periodic activity is proposed, and to completely extract each spectral component both eigen-elements of the respective pair must be used. Conversely, with this proposed technique no detection of pairs of POD modes is required to extract a certain spectral component, which turns out to be very useful for very complex and noisy time-series. Furthermore, with respect to SSA the extracted spectral components are characterized by the same time-length of the source-signal, thus avoiding any loss of samples.

Subsequently, the spectral component related to the POD mode 3 is extracted, i.e. the one corresponding to $f_{2}=60 \mathrm{~Hz}$. This spectral component is characterized by a mean value of 2 and standard deviation of 0.087 , while the mean value of the IF is $59.961 \mathrm{~Hz}$, with a standard deviation equal to 0.025 .

The last component related to the frequency $f_{1}=40 \mathrm{~Hz}$, is extracted by using the POD conv-mode 5 . The extracted component has the following characteristics: modulus with a mean value of 1.003 and standard deviation of 0.091 , mean IF equal to $39.995 \mathrm{~Hz}$, with a standard deviation of 0.053 .

\section{Application of the POD procedure to hot-wire signals}

The procedure based on POD for component detection and extraction from time-series is now applied to the case of hot-wire 
anemometry signals acquired in proximity of the wake generated from a triangular prism.

The wakes produced from finite-length circular cylinders and prisms are dominated by the alternate vortex shedding from the body sides, but further fluctuations at different frequencies, connected with the dynamics of the vorticity structures originated over the body free-end, may appear. Considering circular cylinders, pressure and velocity measurements have shown the presence of clear vortex shedding from most of the cylinder span with Strouhal numbers of the same order as those typical of 2D flows (see e.g. Farivar, 1981; Ayoub and Karamcheti, 1982; Fox et al., 1993). However, a lower frequency was found in a zone approaching the free-end of the cylinders and in Park and Lee (2000) these fluctuations were shown to be associated with a couple of counter-rotating streamwise vortices originating from the model free-end.

Regarding bodies with fixed separation points, the effects of the three-dimensional flow on the vortex shedding generated from triangular plates with different tapers and aspect ratio were investigated in Castro and Watson (2004). An analysis of the mean and fluctuating loads acting on triangular prisms placed with different wind directions was presented in several works like Lindsey (1938), El-Sherbiny (1983), Luo et al. (1994) and Alonso (2005). In Srigrarom and Koh (2008) a qualitative analysis of the flow generated from a rotationally oscillating triangular cylinder was carried out through flow visualizations and PIV measurements. In Iungo and Buresti (2009) the interaction between the alternate vortex shedding and the oscillation of the streamwise vorticity structures generated over the model free-end is investigated, through flow visualizations, velocity and force measurements, by varying the wind direction.

In Buresti and Iungo (2010) it was highlighted that a wake with highly complex morphology and dynamics is produced from a prism with equilateral triangular cross-section orientated with its apex edge against the incoming wind. The model aspect ratio is $h / w=3$, where $h$ is the height and $w$ the base edge of the model, and the tests were carried out at a Reynolds number, based on $w$, of $1.5 \times 10^{5}$. For this configuration flow fluctuations at three prevailing frequencies were singled out, with different relative intensities depending on the wake regions. In particular, the frequency connected with alternate vortex shedding from the vertical edges of the prism was found to dominate in the regions just outside the lateral boundary of the wake at a Strouhal number of about $S t=f w / U_{\infty} \approx 0.16$, where $U_{\infty}$ is the freestream velocity. On the other hand, a lower frequency, at $S t \cong 0.05$, was found to prevail in the velocity fluctuations on the whole upper wake. Simultaneous measurements carried out over the wake of the prism at symmetrical locations with respect to the symmetry plane showed that these fluctuations correspond to a vertical, inphase, oscillation of two counter-rotating axial vortices detaching from the front edges of the free-end. This finding was confirmed by the results of a LES simulation of the same flow configuration, described in Camarri et al. (2006), which also highlighted the complex topology of the upper near-wake produced by the vorticity sheets shed from all the edges of the prism. Wake velocity fluctuations were also observed at an intermediate frequency $S t \cong 0.09$, and were found to prevail in the symmetry plane and may be caused by a flag-like oscillation of the sheet of transversal vorticity shed from the rear edge of the body free-end, and approximately lying along the downstream boundary of the recirculation region in the central part of the near-wake.

The time-frequency analysis of a hot-wire signal acquired in proximity of the wake is carried out and its Fourier power spectrum is reported in Fig. 3(a). The map of the modulus of the wavelet coefficients, calculated through a Morlet function (Fig. 3(c)), shows that a large amount of the energy of this signal is included in a frequency range between $\mathrm{St} \approx 0.03$ and 0.2 ; several spectral components seem to be present but their detection is not sufficiently clear due to their comparable energy and limited spectral separation. However, the wavelet spectrum in Fig. 3(b) highlights the presence of three dominant spectral contributions at $\mathrm{St} \approx 0.05,0.09$ and 0.16 . As observed from the wavelet map, the extraction of these components through bandpass filtering is very challenging because they are highly modulated and spectrally close.

Before performing the spectral decomposition, the signal is filtered through a high-pass filter with a cut-off frequency of St $=0.03$, in a

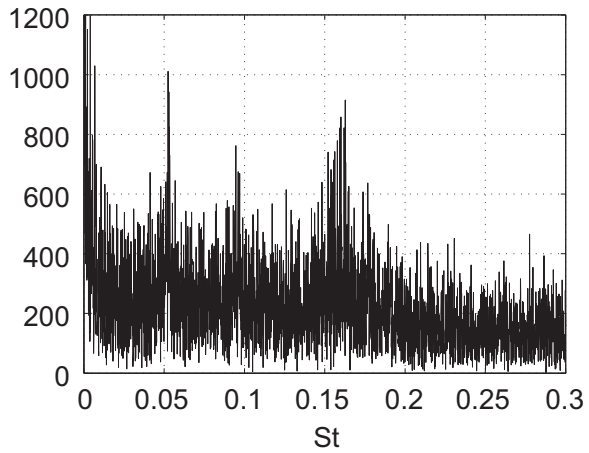

b

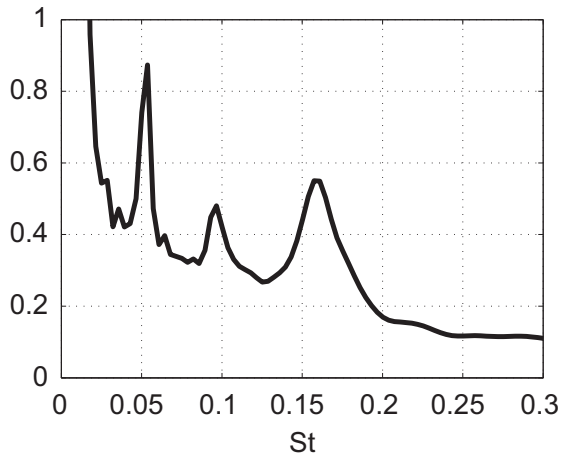

C

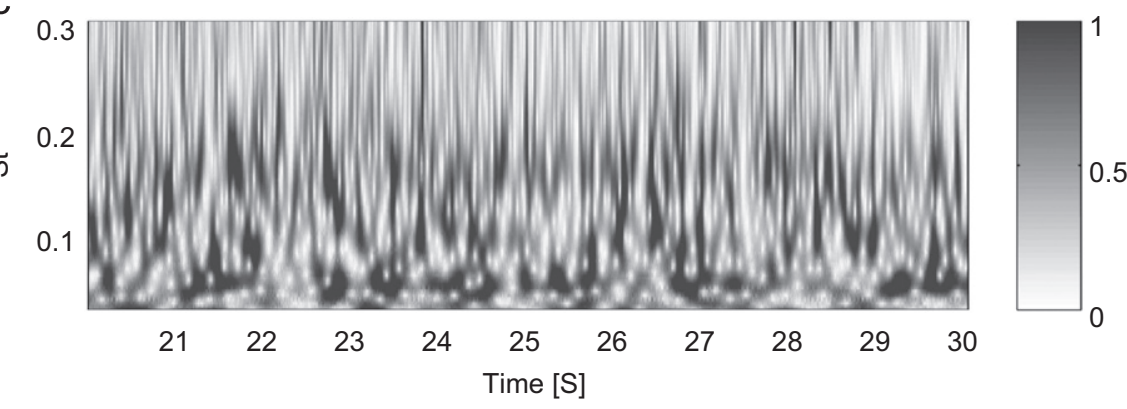

Fig. 3. Hot-wire signal: (a) Fourier power spectrum; (b) wavelet spectrum; (c) map of the modulus of the wavelet coefficients. 
order to remove the typical low-frequency flow fluctuations that were known to be present in the wind tunnel freestream and, thus, avoiding to analyze spectral components with no physical meaning. The POD procedure is applied to the signal by using a number of samples for each snapshot equal to 1001, i.e. a frequency resolution of about $2 \mathrm{~Hz}$; a total number of snapshots equal to $10^{4}$ is produced. The obtained first 50 POD eigenvalues are reported in Fig. 4.

Starting with the extraction of the spectral contribution related to the most energetic POD mode, viz. POD mode 1 , it is seen from its Fourier power spectrum, reported in Fig. 5 that it represents a narrow-band signal at $\mathrm{St} \approx 0.05$. As suggested in Buresti and Iungo (2010), this spectral contribution is connected to the dynamics of a couple of axial vortices detaching over the model free-end. In effect, as this velocity signal was acquired at a relative high position, this phenomenon turns out to be the most energetic one.

The POD mode 2 is the one coupled to the POD mode 1, i.e. characterized by roughly the same power spectrum but with a phase shift of $90^{\circ}$. The spectral contribution extracted with the POD mode 2 from the residual signal, obtained after the extraction of the contribution due to the POD mode 1 , represents a

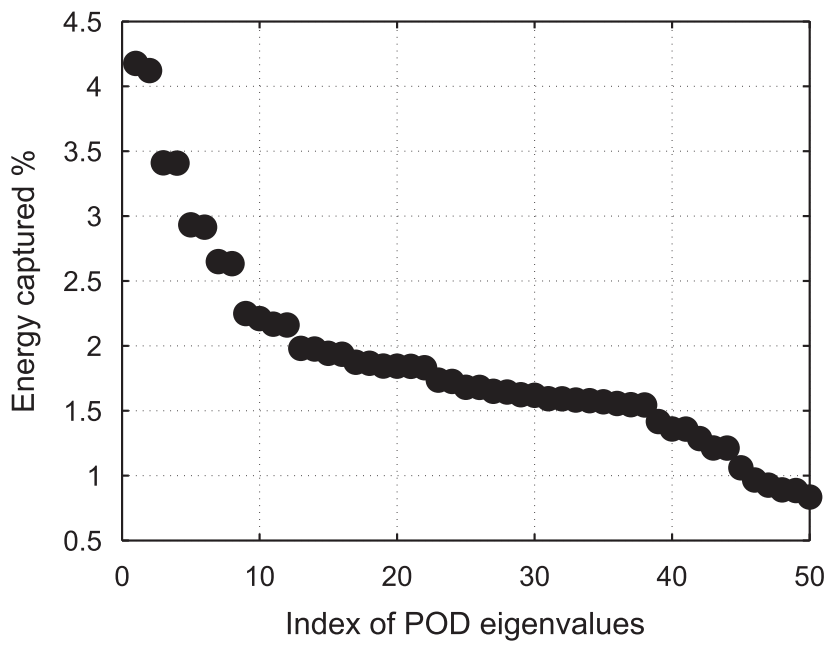

Fig. 4. First 50 POD eigenvalues evaluated for the hot-wire anemometry signal.

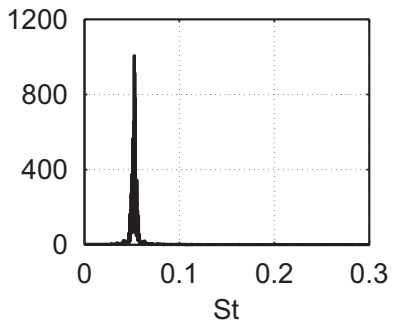

POD mode 1

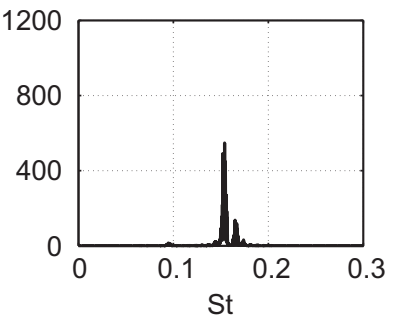

POD mode 5

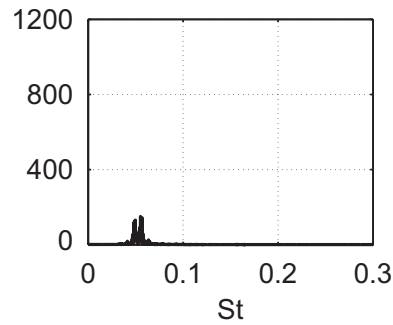

POD mode 2

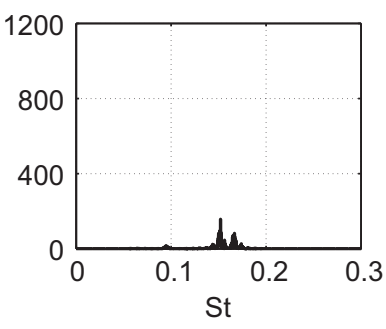

POD mode 6 residue of the contribution previously extracted with the POD mode 1, as shown in Fig. 5.

Moving to the extraction of the spectral contribution connected to the POD mode 3 , the corresponding power spectrum shows that it is characterized by a mean Strouhal number of St $\approx 0.16$, while POD mode 4 is its coupled one.

The following analyzed POD mode 5 represents a further contribution to the spectral component due to alternate vortex shedding being characterized by a mean IF of $\mathrm{St} \approx 0.16$. The POD mode 6 is its coupled one.

Interestingly, the spectral contribution related to the POD mode 7 is characterized by St $\approx 0.09$. POD mode 8 is its coupled one.

Subsequently, the extracted spectral contributions are grouped by their mean IF in order to obtain three different spectral components. Therefore, the first component is obtained by adding the contributions due to the POD mode 1 and 2 , the second with the POD modes from 3 to 6 and the last one with the POD

Table 1

Statistics of the spectral components extracted from the hot-wire signal.

\begin{tabular}{lllll}
\hline POD modes used & Mean modulus & $\sigma$ modulus & Mean IF & $\sigma$ IF \\
\hline 1,2 & 0.09 & 0.05 & 0.052 & 0.004 \\
$3,4,5,6$ & 0.11 & 0.06 & 0.159 & 0.008 \\
7,8 & 0.07 & 0.03 & 0.096 & 0.006 \\
\hline
\end{tabular}

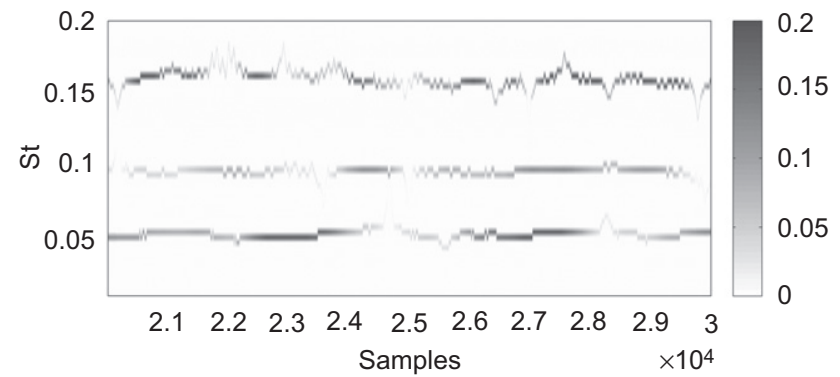

Fig. 6. Hilbert spectrum of the hot-wire signal.
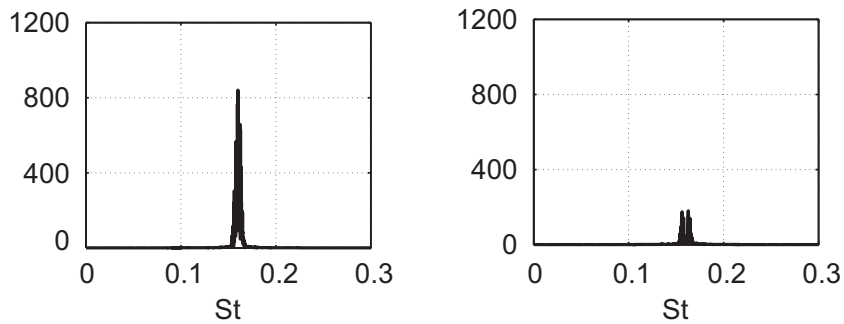

POD mode 3

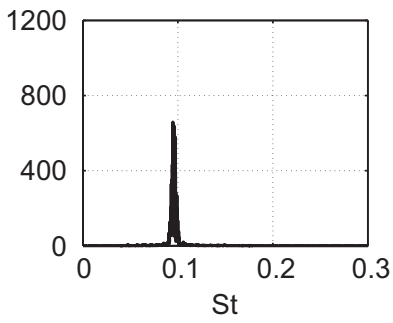

POD mode 7
POD mode 4

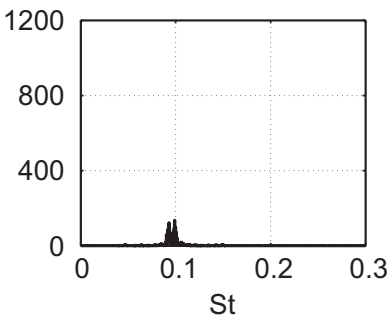

POD mode 8

Fig. 5. Fourier power spectra of the components extracted with different POD modes from the hot-wire signal. 

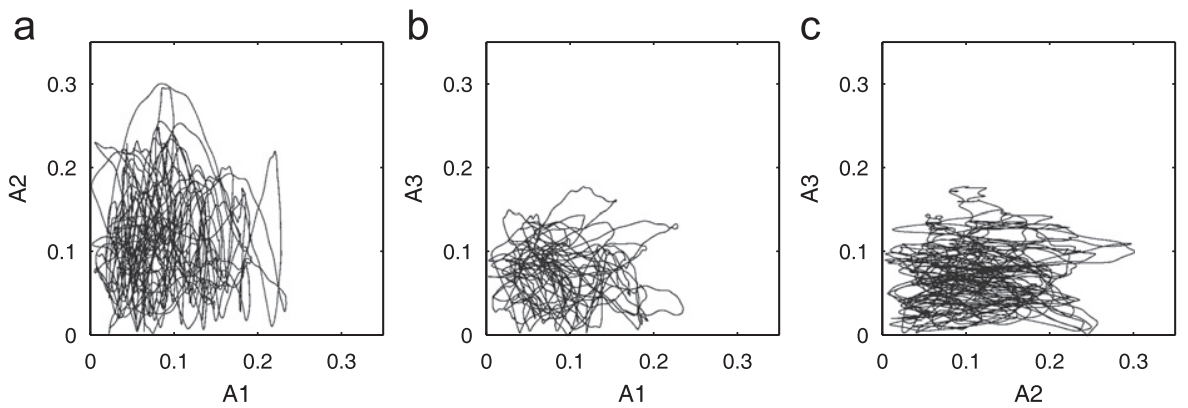

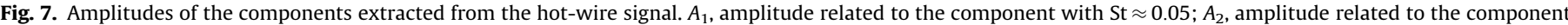
with $\mathrm{St} \approx 0.16 ; A_{3}$, amplitude related to the component with $\mathrm{St} \approx 0.09$. (a) $A_{1}$ versus $A_{2} ;$ (b) $A_{1}$ versus $A_{3}$; (c) $A_{2}$ versus $A_{3}$.

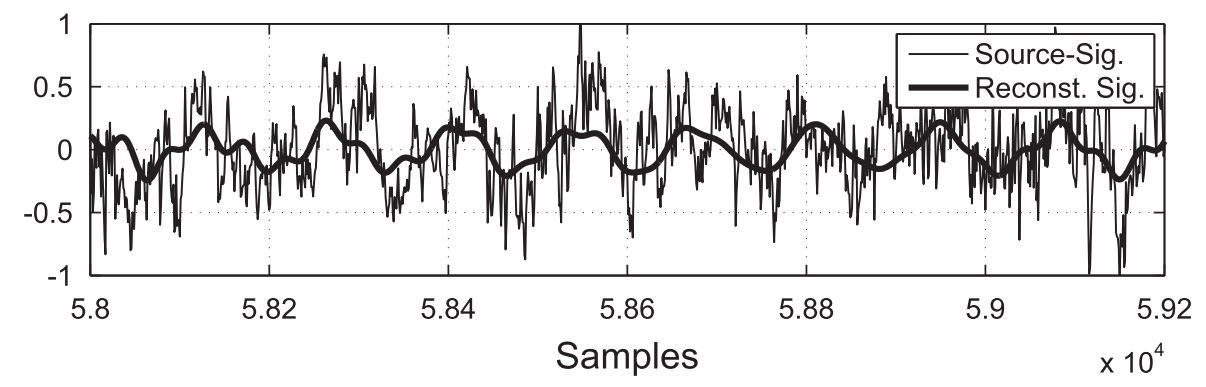

Fig. 8. Reconstruction of the hot-wire signal.

modes 7 and 8 . The statistics of these three spectral components are reported in Table 1 .

In Fig. 6 the resulting Hilbert spectrum (a 3D plot where the $x$-axis represents time, $y$-axis the frequency and grey level the envelope of the extracted components) related to these three components is reported. Those components are found to be practically uncorrelated, as can be seen from Fig. 7. Indeed, the correlation coefficient between the envelope of the component at St $\approx 0.05$ and the one at $\mathrm{St} \approx 0.16$ is $e_{12}=-0.05$, the one between the component at $\mathrm{St} \approx 0.05$ and the one at $\mathrm{St} \approx 0.09$ is $e_{13}=0.01$, and the remaining one $e_{23}=0.01$. Therefore, this can assess that the physical origin of these flow fluctuations is due to the dynamics of different vorticity structures. Finally, in Fig. 8 the reconstructed signal obtained by adding the three spectral components is compared to the source-signal showing that this simplified signal represents the skeleton of the source-signal from which the effects due to turbulence or to instrument noise are removed.

\section{Discussion and conclusions}

A procedure based on proper orthogonal decomposition (POD) for detection and extraction of components present in time-series has been presented. The time-series is divided into different timeportions, denoted as snapshots, uniformly distributed along the signal time-length and composed of the same number of samples. POD is then applied to these snapshots producing an orthogonal basis composed of the so-called POD modes, for which the dominant ones represent the most significant realizations of the analyzed process. The POD modes are sorted by their significance and their contributions to the total energy of the signal is also evaluated.

The extraction of a component corresponding to a certain POD mode is performed through the convolution of the source-signal with the considered POD mode, suitably manipulated. For PODbased techniques a pair of eigen-elements with roughly the same energy is typically detected when a periodic activity is present in the signal and only the sum of the components related to these two eigen-elements enables the reconstruction of the considered spectral component. Conversely, with this convolution procedure a complete component extraction is performed by using only one POD mode of the pair, so that the detection of pairs of eigenelements is not required.

Due to the POD optimality, an automated procedure for principal component extraction is used, and the fluctuations of a signal can be considered as adequately characterized when a certain fluctuating energy is extracted or when the main spectral components are captured.

The procedure based on the POD for component detection and extraction has been applied to hot-wire signals acquired in proximity of the wake generated from a triangular prism placed with a vertical edge against the incoming flow. Flow fluctuations due to the dynamics of different vorticity structures can be easily extracted through the analysis of the most energetic POD modes and the time-frequency analysis is simultaneously carried out through the use of the Hilbert spectrum.

\section{Acknowledgements}

The authors would like to thank G. Buresti, L. Carassale and M.V. Salvetti for their invaluable suggestions.

\section{References}

Alonso, G., 2005. Fenomenos de galope en obstaculos de section no rectangular. Ph.D. Thesis, Universidad Politecnica de Madrid.

Ayoub, A., Karamcheti, K., 1982. An experiment on the flow past a finite circular cylinder at high subcritical and supercritical Reynolds numbers. J. Fluid Mech. $118,1-26$.

Bakewell, H.P., Lumley, J.L., 1967. Viscous sublayer and adjacent wall region in turbulent pipe flow. Phys. Fluids 10, 1880-1889.

Bergmann, M., Bruneau, C.H., Iollo, A., 2009. Enablers for robust POD models. J. Comput. Phys. 228 (2), 516-538.

Buffoni, M., Camarri, S., Iollo, A., Salvetti, M.V., 2006. Low-dimensional modeling of a confined three-dimensional wake flow. J. Fluid Mech. 569, 141-150. 
Buresti, G., Iungo, G.V., 2010. Experimental investigation on the connection between flow fluctuations and vorticity dynamics in the near wake of a triangular prism placed vertically on a plane. J. Wind Eng. Ind. Aerodyn. 98, $253-262$.

Buresti, G., Lombardi, G., Bellazzini, J., 2004. On the analysis of fluctuating velocity signals through methods based on the wavelet and Hilbert transforms. Chaos Solitons Fractals 20, 149-158.

Camarri, S., Salvetti, M.V., Buresti, G., 2006. Large-eddy simulation of the flow around a triangular prism with moderate aspect-ratio. J. Wind Eng. Ind. Aerodyn. 94 (5), 309-322.

Carassale, L., Solari, G., Tubino, F., 2007. Proper orthogonal decomposition in wind engineering. Part 2: theoretical aspects and some applications. Wind Struct. 10 (2), 177-208.

Carmona, R., Hwang, W.L., Torresani, B., 1998. Practical Time-Frequency Analysis. Academic Press, San Diego, CA.

Castro, I.P., Watson, L., 2004. Vortex shedding from tapered, triangular plates: taper and aspect ratio effects. Exp. Fluids 37, 159-167.

El-Sherbiny, S., 1983. Flow separation and reattachment over the side of a $90^{\circ}$ triangular prism. J. Wind Eng. Ind. Aerodyn. 11, 393-403.

Farivar, D.J., 1981. Turbulent uniform flow around cylinders of finite length. AIAA J. $19,275-281$

Fox, A., Karamcheti, T.A., Apelt, C.J., West, G.S., 1993. The aerodynamic disturbance caused by the free-ends of a circular cylinder immersed in a uniform flow. J. Wind Eng. Ind. Aerodyn. 49, 389-400.

Galletti, B., Bruneau, C.H., Zannetti, L., Iollo, A., 2004. Low-order modeling of laminar flow regimes past a confined square cylinder. J. Fluid Mech. 503, $161-170$.

Gamard, S., George, W.K., Jung, D., Woodward, S., 2002. Application of a "slice" proper orthogonal decomposition to the far field of an axisymmetric turbulent jet. Phys. Fluids 14 (7), 2515-2522.

Holmes, P., Lumley, J.L., 1996. Turbulence, Coherent Structures, Dynamical Systems and Symmetry. Cambridge University Press.
Iungo, G.V., Buresti, G., 2009. Experimental investigation on the aerodynamic loads and wake flow features of low aspect-ratio triangular prisms at different wind directions. J. Fluids Struct. 25, 1119-1135.

Lindsey, W.F., 1938. Drag of cylinders of simple shapes. NACA Report 619.

Lumley, J.L., 1967. The structure of inhomogeneous turbulence. Atmospheric Turbulence and Wave Propagation, pp. 166-178.

Luo, S.C., Yazdani, Md.G., Chew, Y.T., Lee, T.S., 1994. Effects of incidence and afterbody shape on flow past bluff cylinders. J. Wind Eng. Ind. Aerodyn. 53, 375-399.

Ma, X., Karniadakis, G.E., 2002. A low-dimensional model for simulating threedimensional cylinder flow. J. Fluid Mech. 458, 181-190.

Olhede, S., Walden, A.T., 2004. The Hilbert spectrum via wavelet projections. Proc. R. Soc. London A 460, 955-975.

Park, C.W., Lee, S.J., 2000. Free end effects on the near wake flow structure behind a finite circular cylinder. J. Wind Eng. Ind. Aerodyn. 88, 231-246.

Pastur, L.R., Lusseyran, F., Faure, T.M., Fraigneau, Y., Pethieu, R., Debesse, P., 2008 Quantifying the nonlinear mode competition in the flow over an open cavity at medium Reynolds number. Exp. Fluids 44, 597-608.

Payne, F.R., Lumley, J.L., 1967. Large eddy structure of the turbulent wake behind a circular cylinder. Phys. Fluids, S194-S196.

Perrin, R., Braza, M., Cid, E., Cazin, S., Chassaing, P., Mockett, C., Reimann, T., Thiele, F., 2008. Coherent and turbulent process analysis in the flow past a circular cylinder at high Reynolds number. J. Fluids Struct. 24, 1313-1325.

Solari, G., Carassale, L., Tubino, F., 2007. Proper orthogonal decomposition in wind engineering. Part 1: a state-of-the-art and some prospects. Wind Struct. 10 (2), $153-176$.

Srigrarom, S., Koh, A.K.G., 2008. Flow field of self-excited rotationally oscillating equilateral triangular cylinder. J. Fluids Struct. 24, 750-755.

Vautard, R., Yiou, P., Ghil, M., 1992. Singular-spectrum analysis: a toolkit for short, noisy chaotic signals. Physica D 58, 95-126.

Weller, J., Camarri, S., Iollo, A., 2009. Feedback control by low-order modeling of the laminar flow past a bluff body. J. Fluid Mech. 634 (2), 405-418. 